\title{
The Possibility of Chinese Characters Becoming Universal Characters From the Perspective of the Development and Function of Chinese Characters
}

\author{
Zhang Ling ${ }^{1,}$ a, Chen Lina ${ }^{2, b}$, Pan Yingdian ${ }^{3, c^{*}}$ \\ ${ }^{1,3}$ School of International Education, Yangtze Normal University, Chongqing, China \\ ${ }^{2}$ Chinese Language \& Culture College, Beijing Normal University, Zhuhai, China \\ a568499822@qq.com \\ blina_ytu2010@163.com \\ cpanyingdian@126.com \\ *Corresponding author
}

\begin{abstract}
Chinese characters are not only the symbolic system of recording Chinese, but also the cultural link of national unity. Chinese characters cannot be assimilated in the ocean of letter characters, on the one hand, due to the barrier of geographical environment, Chinese characters have long enough space for development; on the other hand, Chinese characters have developed from sacrifice and etiquette functions to an effective tool for the national maintenance and unity. The Chinese character standardization measures taken by the past dynasties in China strengthened the management and rule of the country and enhanced the sense of national identity. Through the concrete practice of New China, Chinese characters have been reborn and become the cornerstone of Chinese cultural confidence. With the great rejuvenation of the Chinese nation, Chinese characters will also usher in a new opportunity for development.
\end{abstract}

Keywords: Chinese characters, universal characters, single written Chinese characters

\section{从汉字发展历程与功能看汉字成为世界 通用文字的可能性}

张玲 ${ }^{1 \mathrm{a}}$, 陈黎娜 ${ }^{2 \mathrm{~b}}$, 潘英典 ${ }^{3 \mathrm{c} *}$

${ }^{1,3}$ 长江师范学院国际学院, 涪陵, 重庆, 中国

“北京师范大学汉语文化学院, 珠海, 中国

a568499822@qq.com

blina_ytu2010@163.com

cpanyingdian@126.com

*通讯作者

\section{摘要}

汉字有没有成为世界通用文字的可能？提出这个问题需要勇气更需要自信。与世界上的拼音文字不同， 汉字作为四大古文字硕果仅存的表意文字，不仅是记录汉语的符号系统，更重要的是维护中国统一的 文化纽带。从文字的发展历程来看, 汉字能在字母文字的海洋中不被同化, 一方面是由于地理环境的 阻隔, 使汉字有足够长的发展空间; 另一方面是汉字从祭祀与礼仪功能发展成为国家维护统一的有效 工具。历朝历代采取的汉字规范举措加强了国家的管理与统治，秦始皇 “书同文” 的举措更是有效制 止了汉字的分裂，加强民族的认同感。汉字的生命力极其顽强，即使是在蒙古族建立的元朝以及满族 建立的清朝时失去 “国字” 地位之后依然能反客为主。然而近代留洋派把中国落后的原因归结于汉字, 提出了废除汉字采用拼音文字的主张，经过新中国的具体实践，汉字得以正名，成为中国文化自信的 
基石，随着中华民族伟大复兴，汉字也必将迎来发展的新契机。

关键词: 汉字，世界通用文字，书同文

\section{1. 前言}

汉字有可能成为世界通用文字吗? 一百年以前我 们不敢想这个问题, 一百年以后的今天我们有勇气思考 这个问题。

\section{2. 历史上汉字为什么能连绵不断?}

\section{1. 汉字的发展史}

汉字是记录汉语的符号系统，但汉字不仅仅是一套 记录汉语的符号系统。汉字从甲骨文开始不断地进行着 演变, 在大约六千年的时间里先后经历了甲骨文、金文、 大管、小管、隶书、楷书五个阶段。这一过程以隶变为 分水岭, 汉字发展史可以分为古文字和古文字阶段: 古 文字阶段是商代甲骨文, 周代金文, 六国古文, 大管, 秦代小管。今文字阶段是汉代的隶书（包括草隶），魏 晋至今的楷书（包括行书与今草）。期间无论是改朝换 代还是统一分裂, 记录汉语的符号系统从未被别的文字 符合系统所取代过。汉字虽然形体发生了改变, 但本质 没有变, 一直都是由象形、会意、形声、指示、假借、 转注六种造字法组成的汉字系统。

文字最初的起源大多数都是象形的。世界上四大古 文字: 古埃及的象形文字、古巴比伦的楔形文字、印第 安人的雅玛文字以及古代中国的甲骨文。众所周知, 由 于各种原因, 前三种文字都已经消失在历史的长河中, 只有汉字延续到了现在, 展现了强大的生命力。目前在 世界文字海洋里, 汉字是特殊的存在, 它是唯一的表意 象形文字，其他文字都是表音的字母文字。

那么, 与其他文字相比, 为什么唯独汉字能延续下 来呢? 如果汉字处在其他文明古国的地理位置汉字还 会不会生存下来? 答案是显而易见的一一被其他文字 代替是大概率事件。因为在尼罗河以及幼发拉底河流域 没有一个文明能熔铸青春, 总是在盛极而衰的时候被一 种文明摧毁、代替。部落之间的征伐不断的现象是一种 常态。同样的在甲骨文存在的殷商时期甚至更早的时 期, 部落之间的征伐应该也是这样的, 但为什么甲骨文 没有被另一个部落抛弃呢?

\section{2. 历史上汉字不被其他文字取代的原因}

\section{2. 1. 相对封闭的地理环境为汉字发展提供 了空间}

我们认为地理环境是最重要的因素。世界屋脊青藏
高原是中国最大、世界海拔最高的高原。它南起喜马拉 雅山脉南缘, 北至昆仑山、阿尔金山和祁连山北缘, 西 部为帕米尔高原和喀喇昆仑山脉, 东及东北部与秦岭山 脉西段和黄土高原相接。青藏高原与云贵高原、黄土高 原及其山脉一起构成了中国西南西北一道很难逾越的 巨大防线。中国东部和南部被太平洋的渤海、黄海、东 海、南海包围。这些地理因素阻隔了很多新民族进入东 亚, 导致整个东亚地区处于一个相对封闭的状态, 这种 地理环境对世界文明交流来说是一种阻隔, 但对中国文 明来说却是一种保护。没有这个大环境, 不可能有汉字 的延续性, 也不可能有如此延绵不断的中国文明。在这 种相对封闭的自然环境下, 外族入侵多数是通过北方蒙 古高原南下的草原游牧民族。中国的历史大体是不断的 循环上演的。直到近代工业革命之后航海业兴起, 西方 列强从海上带着坚船利炮而来中国这种相对封闭的环 境才被彻底打破。

\subsection{2. 汉字祭祀与礼仪功能促使汉字保持稳} 定

另外一个导致汉字未被中断的原因恐怕是汉字祭 祀与礼仪的功能了。农业是文明产生非常重要的基础, 流域是农业发展的土壤, 文字是判断文明产生的条件之 一。黄河流域是甲骨文发源的地方。从功能上说, “中 国的汉字起源于神教和占卜，而非经济或者商业原因” 1。从目前我国专家对出土的甲骨文的研究来看, 绝大 多数甲骨文记录的事件都是跟战争、祭祀、占卜有关。 所以从功能上讲, 汉字起源于神教与占卜是正确的。而 在生活在地中海周围, 善于经商的腓尼基人为了更好的 记录与交际在象形文字的基础上创造出了字母, 这些字 母逐渐发展成了希腊字母、拉丁字母, 成为了拼音字母 的源头。《左传》说, “国之大事在祀与戎”。祭祀 (包 括祈祷风调雨顺等农事活动) 、战争对农耕文明的重要 性是不言而喻的。甲骨文的材料显示, 殷商时期, 部落 凡有大大小小的活动都要占卜, 并把占卜的结果刻在龟 甲兽骨之上。占卜这种活动是与鬼神对话, 自然是庄严 神圣的事情。从事这种工作的人一般是部落里掌握占卜 技术与文字的巫师, 这部分人一般是身兼部落的知识分 子、哲学家、思想家、谋士、祭司、文字学家等职位, 有比较高的地位。他们把重要的事件用象形文字 (占多 数) 记录在甲骨之上, 这些甲骨文就成为了被供奉的神 物。文字也变得神秘和神圣起来。在黄河流域生活的各 大小部落面对相同封闭的地理环境和相似的气候, 经过 长期的交流融合, 在类似的生产技术和有限的认知水平 下, 很有可能大多数部落都有相似的活动, 比如祭祀、 战争以及占卜记录等等。文字最早的形式一般都是象形 的, 可以推测黄河流域各部落很可能都有自己的形象文 字。在相似的经济基础与生产条件下, 我们认为当时黄 
河流域不可能只有一种殷商时期的甲骨文，其他形式的 甲骨文或者石刻文也必定存在。

总之, 我们认为汉字之所以未被拼音文字所取代的 原因一是因为地理环境与外界阻隔很大, 这使汉字的源 头甲骨文有足够发展的时间与空间, 免受外来文字的攻 击和取代。二是汉字造字的初衷是记录祭祀与战争, 汉 字作为一种交际工具的存在是次要的, 更多的是代表一 种圣神的权威, 这种权威是跟整个部落的命运联系在一 起的。只要族不灭, 文字就不会灭。特别是在经过数千 年发展之后由汉字形成的瀚如烟海的文献成就, 使得汉 字牢牢地与汉语融为一体了。

\section{3. 汉字是维护国家统一的工具与文化纽带}

\section{1. 汉字与拼音文字相遇}

时到今日, 这两个原因都不复存在了。地理环境方 面, 即使是被少数民族统治, 汉族可凭借先进的文化与 文明不断同化从北方的草原游牧民族及其政权, 汉字历 经几千年而能延续生命。大航海时代来临后, 从海上陆 续有新的民族与文明进入中国版图, 虽然明清两朝有意 进行海禁, 但挡不住时代的潮流, 错过了第一次和第二 次工业革命后的中国已经全面落后与世界强国, 在帝国 主义的坚船利炮下被迫打开国门迎接各方面的挑战, 汉 字命运也面临同样的困境。汉字在功能方面, 早已从记 录 “祀与戎” 进入了广阔的语言文化生活, 在漫长的岁 月中汉字依然没有自然地演化为拼音文字。“中国文献 大量写出之前, 中国人还不知道有简单的字母, 等到知 道的时候, 他们已不愿意放弃长期受到尊重的文字系统 了”2这种说法十分片面, 也站不住脚。在中国漫长的 历史过程中早就接触到了拼音文字只不过非拉丁字母 而已, 比如东汉时期佛经开始传入中国, 那时候中国人 就开始接触梵文并持续了一千多年, 梵文佛经翻译浩如 烟海, 佛教思想更是本土化为中国传统思想之一。即使 是这样, 梵文这种天城体拼音文字从未没有撼动过汉字 的地位。又比如第一个有历史明确记载使用文字的突厥 草原民族与中国隋唐进行过长期的交往与战争。突厥人 在五世纪初就根据古粟特文创立了突厥文, 要是因为汉 字增加了书写与识记的负担就成为抛弃的理由, 那么在 一千多年前就可以采用天城体文或者突厥文了。显然, 中国历朝历代使用汉字的目的并不是用于扫盲。我们认 为中国各朝各代使用汉字的根本原因是用汉字能有效 维护国家的统一。汉字首先是国家的统治工具, 其次才 是交际工具。我们从秦始皇 “书同文” 文字改良运动就 可以看到这一点。

\section{2. 秦始皇“书同文”文字改良运动及其影响}

春期战国末期战国七雄之一的秦国迅速崛起，于公 元前 236 年至公元前 221 年间通过军事与外交战略先后 灭掉了韩、赵、魏, 楚、燕、齐六国, 建立了秦朝一一 中国历史上第一个中央集权封建国家。同时结束了中国 历史上 500 多年的诸侯割据的局面，使中国重新归为大 一统。

在如此长的分裂时间里，使源自夏商周相对统一的 金文在各国逐渐分化出自己的特点, 被学者统称为战国 文字，其中又可以分为 “六国文字” 和 “秦国文字” 两 大系统。前者指除秦国外的六国与其他小国的文字，总 体特点为笔画松散, 随意。后者秦国文字则较多的保持 了西周和春秋时期的金文严谨统一的特点并发展成为 了 “大管” 文。这种体系下的文字一字往往有多种写法, 比如一个 “马” 字, 在当时至少有七种写法, 如图所示:

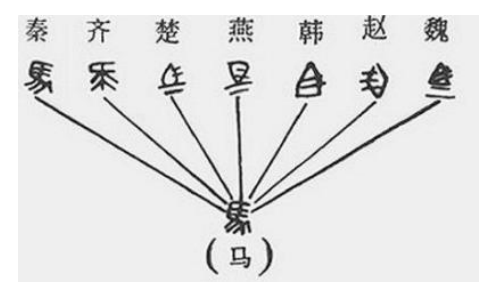

图 1 战国时期马的七种写法

当时的文字乱象，极大地阻隔了政令的通畅与各国 文化的交流以及人民对国家的认同。《说文解字・序》 说 “其后诸侯力政，不统于王，恶礼乐之害己，而皆去 其典籍, 分为七国。田畴异亩, 车涂异轨, 律令异法, 衣冠异制, 言语异声, 文字异形。”为加强帝国的统一, 秦始皇对新帝国进行了文字统一规范。“秦始皇初兼天 下，丞相李斯乃奏同之，罢其不与秦合者。斯作《仓颕 篇》, 车中府令赵高作《爱历篇》, 太史令胡冊敬作《博 学篇》。皆取史篮大管, 或颇省改, 所谓小管也。是时, 秦灭书籍, 涤除旧典, 大发吏卒, 兴戍役, 官狱职务繁, 初有隶书, 以趣约易, 而古文由此而绝矣! ”实际上, 书同文开始推行的时间文献中记载为秦始皇帝二十六 年。但从现实中的实际情况来看, “书同文” 运动早在 秦统一六国之前, 伴随着秦的兼并战争已悄然开始, 特 别是俗体隶书的 “书同文” ，只是未能提到一定的高度 来推行 3 。李斯、赵高等人进行文字统一工作, 在秦国 原来使用的大管籕文的基础上, 进行简化, 取消其他六 国的异体字, 创制了统一文字书写形式, 这就是小管。

“秦焚烧先典，乃废古文，更用八体” 4。“秦焚烧先 典, 乃废古文, 更用八体” 除了小管之外, 大管依旧在 使用, 还有另外六种字体也是秦朝常用的字体分别是: 刻符、摹印、虫书、署书、叒书、隶书。

秦始皇 “书同文” 的影响非常深远。对文字系统本 身而言, 隶变是古今文字的分水岭, 对汉字的发展产生 了深远的影响。《四体书势》说: “隶书者, 管之捷也。” 隶书是从管书的快写中形成的。事实上从考古发现的秦 系文字的资料上看, 战国晚期隶书就开始形成了。 
为什么隶书会在战国后期形成？原因是便捷书写 的需要。问题的关键是这需求背后反映了出了一个非常 重要的信息: 由于大量记录的迫切需要，推动了汉字的 隶变, 汉字使用率大大提高, 使用范围更加广阔, 促使 汉字从“术业有专攻”的神坛走向语言生活的各个方面。 就文字的统一而言，“书同文” 是一场文字改良运动， 继承了金文的大统, 去伪存真, 摒弃了不合秦国小管标 准的字体, 同时也吸取了一些国家字体的优点 (比如楚 国字体），统一与规范了全国标准用字，及时制止了汉 字继续走向分化, 演化为其它文字的危险, 有效维护了 汉字的稳定。

“书同文”的影响不仅仅在文字系统本身，更重要 的作用是有效地维护了国家统一。首先汉字强大的表意 不表音的功能, 使汉字跨越地域时空的限制, 成为了拥 有各地不同方言语音人们的最大公约数。在没有殊音的 差别下, 每一个地域的方言都可以把汉字作为自己的文 字, 增强了民族凝聚力。表意的汉字不注重承载语音, 而更注重承载意义。规范后汉字更顺畅地将国家的政 令、文化思想、经济、军事等方面法规等传递到全国各 地, 为国家的统一、经济的发展、文化的繁荣都有巨大 的贡献。特别是秦以后, 两汉四百多年皆沿袭秦始皇的 “书同文” 政策。两汉学者用隶书整理浩如烟海的先秦 文献资料, 撰写了不计其数的历史与文学作品, 奠定了 中国文化、制度等各方面的基础。

\section{3. 唐明两朝文字规范措施进一步巩固了汉 字的地位}

事实上, “书同文” 自古有之, 并不是秦始皇的首 创，“书同文” 是历朝历代的国策。秦以前，有 “仓颕 造字”。荷子认为: “故好书者众矣, 而仓颕独传者, 壹也。” 《汉书・古今人表》说: “仓颕, 黃帝史。” 马执斌认为 “仓颕应该是古代中国统一文字的第一人。 西周《周礼・春官 - 外史》有 “掌达书名于四方。，《周 礼・秋官 - 大行人》: “七岁属象胥、谕言语、协辞命, 九岁属韾史、谕书名, 听声音 5。, ”秦以后, “唐太 宗的经书规范举措, 对 《五经定本》与《五经正义》的 编撰, 实现了前所未有的经学读本和经学释义的统一和 规范 6。” 明朝太祖的语言改革和语音规范措施, 明朝 灭亡蒙古人建立的元朝后, 分别采取 “去蒙复华” 的语 言改革, 并命人参照唐宋 “中原雅音标准” 编写《洪武 正韵》确定当时汉语正音标准与文字规范标准, 为正统 华夏语言文字传统的延续和规范产生了深远的影响。由 此可见, 历朝历代都会对汉字进行规范, 汉字问题不仅 仅是汉语言的工具，还是国家统治的基础工具。

以上我们回顾了汉字作为国家统治工具的规范史。 那么现在及未来汉字有没有可能被拼音文字取代? 我 们认为汉字最难的时代已经过去, 以前未被取代, 以后 更不可能会被拼音化或者主动拼音化。
从理论上来汉字拼音化最佳时期是元朝与清朝。这 两个朝代分别由蒙古族与满族建立, 他们都分别将自己 民族的语言与文字以法规的形式定为“国语”和“国字”。

\section{4. 元清两朝的 “国字”政策未能撼动汉字的 地位}

1271 年忽必烈击败阿里不哥后夺得汗位, 建立了一 个庞大的元帝国，此时由成吉思汗建立的大蒙古帝国实 际已经分裂为元朝、钦察汗国、察合台汗国、窝阔台汗 国、伊利汗国五个国家，元帝国只是名义上的大蒙古帝 国的代表。在元朝统治的广哀大地上有多种语言与文字 并存。元帝国成立之初, 忽必烈就认识到全国文字错综 复杂, 传统回鹘体蒙古文标音不太准确, 且是从外族借 用过来的文字, 大元帝国的武功不相匹配, 需新创文字 以显帝国气象。《元史》卷二百二《释老传》: “朕惟 字以书言, 言以纪事, 此古今之通制。我国家肇基朔方, 俗尚简古，未遑制作，凡施用文字，因用汉楷及畏吾字， 以达本朝之言。考诸辽、金, 以及遐方诸国，例各有字， 今文治浸兴, 而字书有阙, 于一代制度, 实为未备。故 特命国师八思巴创为蒙古新字, 译写一切文字, 期于顺 言达事而已。自今以往, 凡有芼书颁降者, 并用蒙古新 字，仍各以其国字副之。”

仅从语言规范的角度上看这是中国历史上继秦王 朝书同文之后的第二次文字大统一的伟大举措, 具有非 常重大的历史意义。八思巴文是一套拼音系统, 相当于 现在的国际音标, 它的优势十分明显, 若能广泛应用, 就可以实现文字大一统。但是八思巴文自 1269 年开始 推广使用到被弃置不用历时仅 100 年左右。一般认为推 广失败的原因主要是八思巴文字系统难记难写。蒙古人 当时已使用回鹘体蒙古文多年，不愿学习难以辨认的八 思巴文; 习惯了使用汉字的汉人更是看不懂也读不出; 藏人习惯横写, 读八思巴文觉得非常别扭, 因此八思巴 文的推广遇到了很大困难。虽然忽必烈于 1271 年下诏 要求百官百日内学会八思巴文, 官小的学会了可以升 官, 没官的学会了给官, 不识字的学会了可以终身免除 乌拉徭役, 但也未能奏效。八思巴文自己本身都没有站 位脚跟, 更不谈不上取代汉字了。加上当时汉字代表的 是更先进的文明与文化，在考虑到民族和谐问题，如果 废除汉字只能是激化民族矛盾，加速王朝的灭亡。

清朝也非常注重强化满文为 “国书”。清朝太祖努 尔哈赤借用回鹘体蒙文字母创制了满文, 皇太极用加圈 点的方式改进了满文并正式将新满文定位为 “国书”。 顺治、康熙、雍正三朝采用强化 “国语”, 满汉合璧, 多语言并用的政策，“到乾隆时期 “国语”和 “汉语” 的使用广度与深度, 均达到前所未有的高度。7”但乾 隆后期至嘉庆与道光时期, 满语与满文迅速衰退, 随着 清朝的衰落与覆灭到现在满语与满文逐渐废弃不用。

元清两种文字都属于拼音文字，在中国取得统治地 位后都极力推广自己的文字，到最后不但没有成功反而 
消亡了。这说明了汉字有强大的抗融能力, 在拥有庞大 的使用人群作为土壤, 以及有文化的先进性加持, 其他 文字进入汉字系统就如泥牛入海般被同化了。

\section{4. 汉字的危机与新生}

堡垒最容易从内部攻破。汉字最困难的时候并不是 元清时期失去统治地位的时候, 而是民国时期至新中国 建国初期, 中国人自己此起彼伏废除汉字的呼声, 差点 自毁长城, 把汉字扔进历史的垃圾堆里。

从明末清初开始中国逐渐落后于西方列强, 以至于 出现了一系列丧权辱国的事件。清末民初中国大量有志 之士走出国门以寻求富国图强的办法, 经过一系列的探 索与尝试都未曾使中国摆脱落后挨打的局面。在民族主 义思潮与救国图存紧迫性的大背景下, 当时主要以海归 派为代表的学者把国家落后的原因归各于记录与传承 中国传统思想的汉字。提出了废除汉字改用拉丁文的主 张, 甚至主张采用世界文字, 其中不乏响当当的人物, 比如吴稚晖、钱玄同、陈独秀、吴玉章、鲁迅、刘半农、 㫿秋白、蔡元培等。言辞之激烈, 现今读起来仍然可以 感觉到当时救国图存的紧迫感, 比如, 鲁迅: “汉字不 灭, 中国必亡!”, 刘半农: “汉字不灭则中国新文化 无望。” 等。与此同时, 以本土派为代表的学者从各个 角度极力反对废除汉字, “中国自古以来, 亡国之祸叠 见, 均国亡而学存。至于今日, 则国未亡而学先亡。竟 亡于教育普兴之世, 不亦大可哀邪！故国学之厄, 未 有甚于今日者也。 8 ” 指出了废除汉字与国家存亡的关 系, 废除汉字是一种自我亡国的体现。章太炎、刘师培 都很强调汉字的不可替代、不可或缺的承载民族历史和 文化的功能, 强烈发对废除汉字。先前主张废除汉字的 吴稚晖也转变了看法, 认为汉字 “必不能废, 摩登学士 想用字母造拼音汉文, 皆不过陃与妄耳 9 ”。

实践是检验真理的唯一标准。汉字拼音化早从明清 传教士学习汉语时发端。1931 年《中国拉丁化字母》出 台, 宗旨是根本废除汉字, 实现纯粹的拼音文字。民国 政府也作出过种种简化汉字和汉字拼音化的努力。但由 于时局动荡, 无力实施而作罢。新中国成立后提出先简 化汉字, 再实现拼音化的目标。而在实践的过程中, 发 现拼音文字不适合拼写汉语, 最直接的原因是拼音文字 不能区分汉语中大量的同音字, 实现拼音化必定会带来 语言的混乱。所以汉语拼音只能作为辅助汉字发音的工 具。基于这种事实, 汉字拼音化遂被放弃。我们认为根 本的原因是新中国成立后在中国共产党的领导与治理 下, 中国经济快速发展、军事实力增强在中华民族伟大 复兴的道路上, 人们发现汉字不仅不是 “落后和野蛮的 文字”, 而是中国文化自信的基石, 是维护国家文化统 一的纽带。

\section{5. 汉字未来有可能成为世界通用文字}

现在我们的问题是汉字在未来能否像拉丁文字一 样成为世界通用文字? 甚至有没有可能超越和替代拉 丁文成为世界通用文字?

看一种文字能否成为世界通用字, 主要有两个标 准。第一个标准是文字本身有没有这个潜质。从汉字的 使用基数来看, 目前除中国大陆外, 中国台湾、香港、 澳门、日本以及海外华侨聚集区都在使用汉字, 加上世 界各地学习汉语的人数约 1 亿人, 保守估计使用人数不 低于 16 亿, 约占世界 76 亿人口的 $21 \%$, 使用基数十分 庞大。从作为通用字的历史来看, 如果把中国看作是一 个小世界, 汉字已经成为了这个小世界的通用文字。不 同的方言之间, 用同一套汉字, 包括中国台湾与香港用 的繁体字都是一脉相承的。在少数民族地区汉字也是作 用通用文字在使用。在整个东亚, 汉字的辐射力也非常 强大, 汉字曾经是日本、朝鲜、越南的国字, 形成了东 亚汉字文化圈, 事实证明汉字可以成为其他语言文化的 载体, 所以汉字是完全有潜质成为世界通用文字的。

第二个标准是这种文字所属的国家是否有足够强 大能为文字提供足够大的市场。国家的经济是否发达, 军事实力是否强大, 文化是否令人向往, 特别是科技是 否发达是决定一个国家是否有能力的关键因素。自明末 清初至今, 中国一直在追赶西方的脚步。今天中国刚摆 脱落后挨打、贫穷孱弱的局面, 经济、军事实力等硬实 力迅速增强, 但仍然处于发展中国家, 大多数领域仍然 需要向强国学习。特别在科技领域, 如今科技是第一生 产力, 但几乎所有自然科学与尖端科技都是以拉丁文特 别是英语字母书写与表达的。全球大约有 3.75 亿人的 第一语言是英语; 另外有大约 3.75 亿人将英语作为第 二语言。世界上大约还有 7.5 亿人将英语作为外语使用 和掌握, 英语是世界上各大航空公司和国际商业界使用 最为广泛的语言。世界上超过 $80 \%$ 的电子储存信息是用 英文写成的, 世界上超过三分之二的科学家能够阅读英 文。世界上有 75 个国家将英语作为官方语言或者给予 英语十分特殊的地位 10。英语俨然已经是世界通用语, 拉丁字母则似乎已经是世界通用文字了。

那么汉字还有没有逆袭的机会? 我们对此持乐观 态度, 虽然汉语有书写困难的问题, 但在信息时代这个 问题已经显得不是那么重要了。汉字有非常重要的一大 优点就是超越地域与语音的表意功能与符号功能, 这套 符号系统与交通符号有相似性, 世界的不同民族可能语 言不同, 不能相互通话, 但是通过学习都可以看懂汉字 表达的意思。这是任何拼音文字都不能比拟的。汉字系 统里的每一个汉字并不是随意组合而是有理据的, 比如 从偏旁就可以猜出汉字的大概意思, 如, “打、找、挂” 都是用手做某事的动词; 现代化的汉字也具有一定的表 音功能, 如, “请、晴、清、精” 都是 “青” 的音变; 加上汉字已经吸收了拉丁字母作为拼音辅助, 最大限度 地弥补了自身的短板。汉字已经为成为世界性通用文字 做好了准备。虽然就目前而言还看不到任何希望, 但是 
随着中华民族的伟大复兴中国梦的实现, 汉字也必将迎 来发展的新契机。

\section{项目基金}

本文为重庆社会科学规划博士项目《基于 “一带一路” 语言战略视野下的汉语与蒙古国 蒙语词汇接触研究》（2018BS34）的阶段性成 果之一。

\section{Reference}

[1] Claude, H. (1999) Verbal activity and talent. In: Haibing, Y. (Eds.), The Man of Words - Linguistic Contribution to the Humanities. SDX Joint Publishing Company, Beijing. pp. 15.

[2] Claude, H. (1999) Verbal activity and talent. In: Haibing, Y. (Eds.), The Man of Words - Linguistic Contribution to the Humanities. SDX Joint Publishing Company, Beijing. pp. 112.

[3] Yu, W. (2014) The new Viewpoint about "Single written Chinese Character" in Qin dynasty. J. Art Education, 2:126.

[4] Jian, X. (2014) The Record of Primary Learning. ZHONGHUA Book Company, Beijing.

[5] Ma, Z.b. (2015) Recognition about Single written Chinese Character. J.The Journal of Handan college, 4:75-79.

[6] Zhou, Q.s. (2017) The Tradition of Chinese Linguistic Culture and the Change of Ancient Language Policy. J.The Study of Language Strategy, 5:72-78.

[7] Zhou, Q.s. (2017) The Tradition of Chinese Linguistic Culture and the Change of Ancient Language Policy. J.The Study of Language Strategy, 5:79.

[8] Wang, L.m. (2017) Language Reform in Late Qing Dynasty. J. Nationalism and Cultural Reform. Academic Monthly, 11:131.

[9] Wang, L.m. (2017) Language Reform in Late Qing Dynasty. J. Nationalism and Cultural Reform. Academic Monthly, 11:132.

[10] Fang, Z.q. (2007) Cognitive Insight into Regional Varieties of English. J. Journal of Ningbao Institute of Education, 4:58-61. 